\title{
Versorgungsplanung \\ am Beispiel der Spitallisten der beiden Basel
}

Michael Steiner

Inhaltsverzeichnis

12.1 Einleitung - 204

12.2 Ausgangslage -205

12.3 Spitalplanung im gemeinsamen Gesundheitsraum (GGR) - 208

12.3.1 Ein Baustein der Spitalplanung - bedarfsgerechte Leistungsmengen - 208

12.3.2 Was bedeutet diese Erkenntnis für die gemeinsame Versorgungsplanung in der Akutsomatik? - 214

12.3.3 Das bedarfsorientierte Planungsmodell - technische Umsetzung - 216

12.4 Das Planungsmodell setzt die Leitplanken für die Spitalliste - 221

Literatur - 221 


\section{- Zusammenfassung}

Mit der bedarfsorientierten Spitalplanung anhand der Nomenklatur der Spitalplanungsleistungsgruppen (SPLG) steigen die Kantone Basel-Landschaft und Basel-Stadt in eine umfassende Versorgungsplanung in der Region Basel ein. Der gesamte Planungsprozess von der Bedarfsanalyse über die Mengenprognose pro SPLG bis hin zur technischen Umsetzung des Planungsmodells anhand einer linearen Optimierung ist Gegenstand dieses Beitrags. Dabei werden die Umsetzungschancen aufgezeigt, die eine medizinisch-planungsorientierte Nomenklatur für die Weiterentwicklung der Krankenhausplanung in Deutschland bieten kann.

With demand-oriented hospital planning based on the nomenclature of hospital planning service groups (SPLG), the Swiss cantons of Basel-Land and Basel-Stadt are starting a comprehensive health care supply planning process in the Basel region. This paper describes the entire planning process, from the analysis of demand and volume prognosis per SPLG to the technical implementation of the planning model along the lines of a linear optimisation. It outlines the opportunities that a planning-oriented medical nomenclature can provide for the further development of hospital planning in Germany.

\subsection{Einleitung}

Wie am Beispiel der Krankenhausversorgung des Landes Nordrhein-Westfalen wird schon seit vielen Jahren eine Konsolidierung der Versorgungsstrukturen auch in der Schweiz angemahnt. Zahlreiche Experten gehen davon aus, dass eine besser abgestimmte Krankenhausversorgung sowohl die Versorgungsqualität erhöht als auch den Anstieg der Kosten dämpfen kann. Vor dieser Herausforderung stehen auch die Kantone Basel-Stadt und Basel-Landschaft.
Mit der Vorstellung des Gutachtens „Krankenhauslandschaft NRW" im Auftrag des Ministeriums für Arbeit, Gesundheit und Soziales in Nordrhein-Westfalen (MAGS 2019) wird genau dieser Fragestellung im Rahmen von Versorgungsanalysen nachgegangen: Gibt es eine Möglichkeit, die Versorgung in NRW effizienter zu gestalten und dabei die Qualität der Versorgung weiterhin hoch zu halten? Dabei nimmt das Gutachten Bezug auf die Planungsgrundlagen der sogenannten Zürcher Leistungsgruppensystematik (Gesundheitsdirektion Zürich 2020). Die Leistungsgruppensystematik mit Spitalplanungsleistungsgruppen (SPLG) bildet die methodische Grundlage für die im Gutachten vorgestellten Versorgungsanalysen. Im Rahmen der Erstellung der sogenannten gleichlautenden ${ }^{1}$ Spitallisten der Nordwestschweizer Kantone Basel-Landschaft (BL) und Basel-Stadt (BS) wird die Leistungsgruppensystematik in Kombination mit der Versorgungsplanung angewendet. Mit dem Planungsmodell, das verschiedene Planungsinstrumente kombiniert, stoßen die beiden Basler Kantone auf schweizweites Interes$\mathrm{se}^{2}$.

Die folgenden Ausführungen stellen das Planungsmodell und die methodischen Grundlagen der Bedarfsprognose vor. Das Planungsmodell ist ein Tool, das den eigentlichen Planungsprozess (- Abb. 12.1) technisch unterstützt.

Mit einer kurzen Einführung zur Versorgungslandschaft der Nordwestschweiz wird die Ausgangslage zur Spitalplanung erläutert.

1 Rein rechtlich erlässt jeder Kanton eine Spitalliste. Die Spitallisten der Kantone Basel-Stadt und BaselLand stimmen hinsichtlich der Leistungsaufträge der Spitäler zukünftig überein.

2 Mehr zum gemeinsamen Versorgungsraum unter: www.chance-gesundheit.ch. 


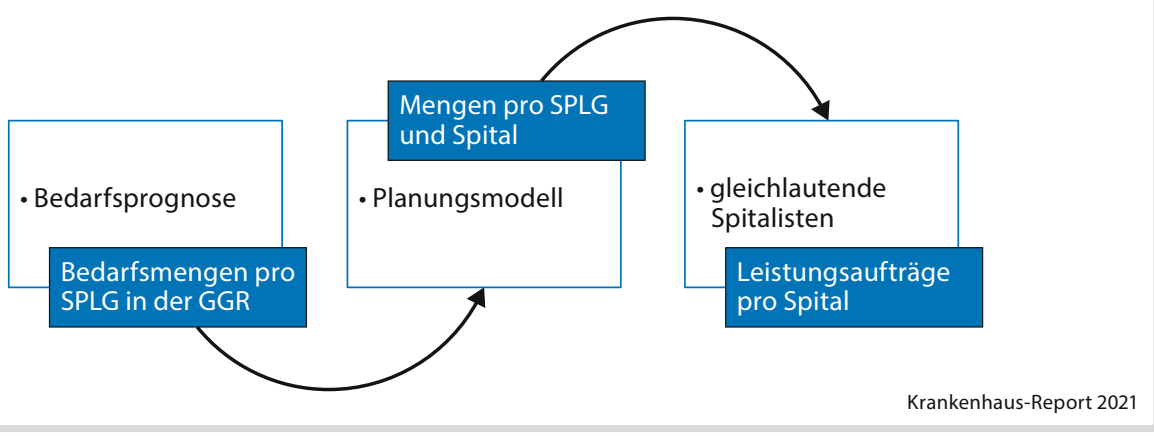

- Abb. 12.1 Der Spitalplanungsprozess

\subsection{Ausgangslage}

Die Region des Jura-Nordbogens kann als ,,integraler Gesundheitsraum" für das gesamte medizinische Leistungsspektrum sowie für alle Bevölkerungsgruppen und Altersstufen betrachtet werden. Die Menschen, die in dieser Region wohnen, lassen sich auch dort behandeln.

Die akutstationäre Versorgungsplanung wurde in der Region bisher von jedem Kanton separat durchgeführt. Mit dem am 10. Februar 2019 vom Stimmvolk der Kantone Basel-Stadt und Basel-Landschaft angenommenen Staatsvertrag zur Planung, Regulation und Aufsicht in der Gesundheitsversorgung erhalten die beiden Kantone die Möglichkeit, die Versorgungsplanung nun gemeinsam durchzuführen.

Ein Beweggrund des Stimmvolks ist sicherlich die Prämienlast, die insbesondere die breite Mittelschicht trifft: Die mittlere Krankenkassenprämie pro Kopf und Monat (- Abb. 12.2) für die beiden Basler Kantone (rote Säulen) gehört zu den höchsten in der Schweiz.

\section{- - Die Bevölkerung wird durch ein dichtes Netz an stationären Angeboten versorgt}

Für die rund 481.000 Personen in den beiden Basel wird die stationäre Versorgung durch 26 Spitalstandorte sichergestellt. Die Spitalstandorte konzentrieren sich im Stadtgebiet
Basel und im Bezirk Arlesheim des Kantons Basel-Landschaft (- Abb. 12.3).

Die Bevölkerung der beiden Basler Kantone lässt sich im Fall eines notwendigen akutstationären Aufenthalts zum weit überwiegenden Teil in einem der Spitäler in einem der beiden Kantone behandeln (2016: 93,2\%). Unter Berücksichtigung der Spitäler in Dornach und Rheinfelden erhöht sich der Anteil auf rund $98 \%$ (Socialdesign 2018). Somit werden Patientinnen und Patienten aus dem gemeinsamen Gesundheitsraum (GGR) überwiegend in Spitälern behandelt, die über einen Leistungsauftrag der Kantone Basel-Stadt und Basel-Landschaft verfügen. Der Abdeckungsgrad der beiden bisher getrennten Spitallisten ist somit sehr hoch.

Eine gemeinsame Spitalplanung bedarf gemeinsamer Versorgungsziele, an denen sich die Leistungsaufträge der Spitäler auszurichten haben, und einer gemeinsamen Nomenklatur, anhand derer die medizinischen Leistungen klassifiziert werden. Die Versorgungsziele leiten sich aus den übergeordneten politischen Zielen des gemeinsamen Gesundheitsraums $\mathrm{ab}$ :

- eine optimierte Gesundheitsversorgung der Bevölkerung der beiden Kantone,

- eine deutliche Dämpfung des Kostenwachstums im Spitalbereich sowie

- eine langfristige Sicherung der Hochschulmedizin in der Region. 


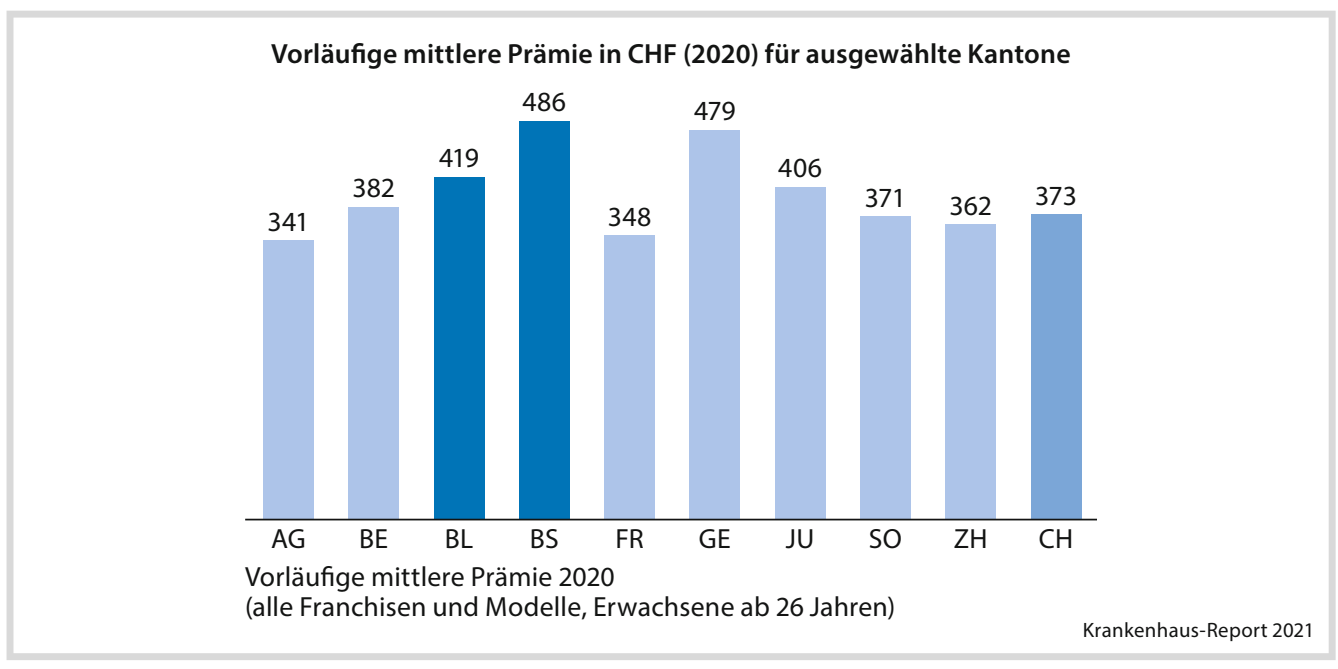

- Abb. 12.2 Mittlere Krankenkassenprämie in beiden Basel und ausgewählten Kantonen (Datenquelle: BAG 2020; eigene Darstellung)
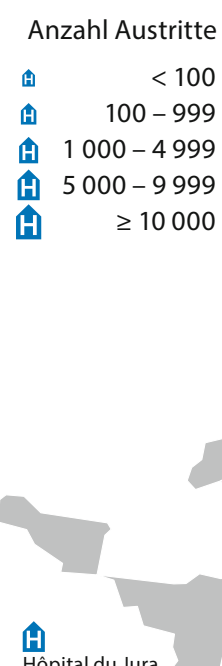

Hôpital du Jura

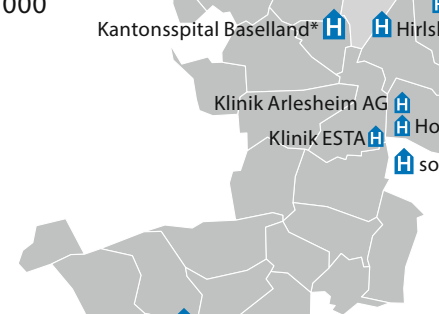

H Kantonsspital Baselland*

$5 \mathrm{~km}$

*Kantonsspital Baselland: Keine Einzelwerte dargestellt

Wert entspricht jeweils dem Mittelwert (ca. 6600 Austritte) der drei Standorte Bruderholz, Liestal und Laufen

**Adullam-Spital: Keine Einzwelwerte dargestellt.

Wert entspricht jeweils dem Mittelwert (ca. 380 Austritte) der beiden Standorte Basel und Riehen

Krankenhaus-Report 2021

- Abb. 12.3 Spitalstandorte im gemeinsamen Gesundheitsraum (GGR), Statistisches Amt Basel-Stadt (2019). (Steiner et al. 2019) 


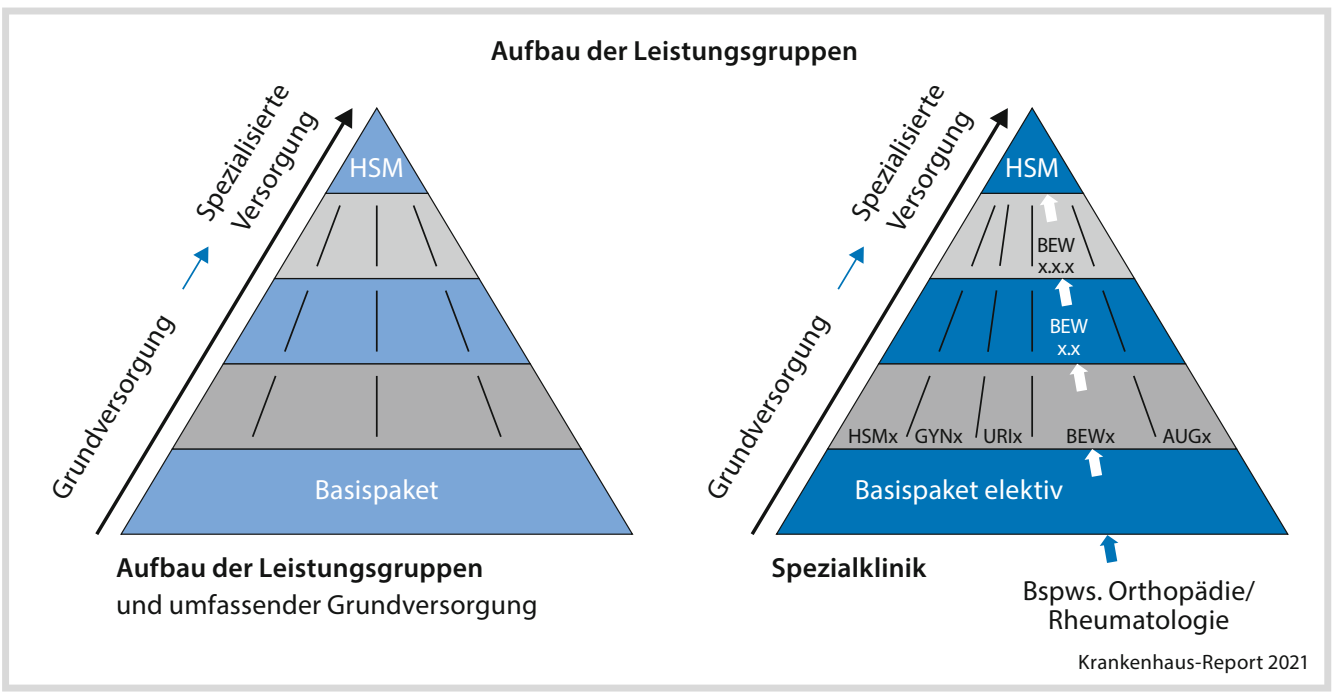

- Abb. 12.4 Hierarchie der Spitalplanungsleistungsgruppensystematik (SPLG) (eigene Darstellung in Anlehnung an Lehmann 2011)

In der Akutsomatik lassen sich die einzelnen medizinischen Behandlungen gemäß der Zürcher Leistungsgruppensystematik (Gesundheitsdirektion Zürich 2020) in 31 klinische Leistungsbereiche einteilen. Damit wird das gesamte akutsomatische Behandlungsspektrum systematisch beschrieben.

Die Klassifizierung fasst DRGs bzw. Diagnose- (ICD) und Operationscodes (CHOP) in medizinisch und ökonomisch sinnvolle Leistungsgruppen zusammen, die hierarchisch aufgebaut sind (- Abb. 12.4). Auf den Leistungen der Grundversorgung bauen Leistungen der erweiterten Grundversorgung und der spezialisierten Versorgung auf. Das sogenannte Basispaket (BP) ist für alle Spitäler mit einer Notfallstation obligatorisch. Das Basispaket ist die Voraussetzung für alle Leistungsgruppen mit einem hohen Notfallanteil, da Notfallpatienten mit häufig unklaren Beschwerden eine breite Basisversorgung benötigen. Eine umfassende Differenzialdiagnostik und sofortige Erstbehandlung soll in jedem Fall möglich sein.

Mit dem Basispaket elektiv (BPE) sichern Spitäler ohne Notfallstation die Grundversor- gung in ausgewählten Leistungsbereichen. Für die Sicherung der Notfallversorgung reicht das Basispaket elektiv nicht aus; es findet somit Anwendung in Spezialkliniken, die sich auf wenige ausgewählte Leistungsbereiche konzentrieren (Bewegungsapparat, Augenheilkunde, Gynäkologie, Urologie, Hals-Nasen-Ohrenheilkunde).

Für jede Leistungsgruppe werden leistungsspezifische Anforderungen und Verknüpfung zwischen den Leistungsgruppen definiert. Leistungsgruppenspezifische Anforderungen können sein:

- Facharzt und Erreichbarkeit

- Notfallstation - Level

- Intensivstation - Level

- Verknüpfte Leistungen (inhouse oder mittels Kooperation)

- Tumorboard

- Mindestfallzahlen

- Sonstige Anforderungen (bspw. Zertifizierungen)

Diese Anforderungen müssen von dem Spital erfüllt werden, das eine entsprechende Leistungsgruppe abbildet. Die Verknüpfung zwi- 
schen den Leistungsgruppen ist medizinisch indiziert.

\subsection{Spitalplanung im gemeinsamen Gesundheitsraum (GGR)}

Die Spitalplanung in der Schweiz erfolgt zunehmend abgestimmt zwischen den Kantonen und gemäß den Empfehlungen der Gesundheitsdirektorenkonferenz. Das Vorgehen der Kantone Basel-Stadt und Basel-Landschaft stützt sich darauf und entwickelt das Vorgehensmodell weiter (GDK 2018).

Die bedarfsgerechte Leistungsmenge bildet die Basis des Prognosemodells ( Abschn. 12.3.1). Die bedarfsgerechte Leistungsmenge pro Spitalplanungsleistungsgruppe (SPLG) ist die um den Korrekturfaktor (angebotsinduzierte Nachfrage) adjustierte Leistungsmenge aus dem Datensatz der medizinischen Statistik $^{3}$ des Bundesamtes für Statistik.

Mithilfe der demographischen Daten können für das Jahr 2018 die Hospitalisierungsraten nach Alter und SPLG berechnet werden. Diese werden anschließend auf die aktuellsten Bevölkerungsprognosen angewandt, um die Anzahl der bis zum Jahr 2028 prognostizieren Fälle zu erhalten.

\subsubsection{Ein Baustein der Spitalplanung - bedarfsgerechte Leistungsmengen}

Mit dem politisch vorgegebenen Ziel, Über-, Unter- und Fehlversorgung zu vermeiden, setzt sich der Versorgungsplanungsbericht $2019^{4}$ intensiv auseinander. Auslöser ist eine Analyse des Schweizerischen Gesundheitsobservatori-

3 https://www.bfs.admin.ch/bfs/de/home/statistiken/ gesundheit/erhebungen/ms.html.

4 https://chance-gesundheit.ch/de/heute. ums (OBSAN) im Rahmen des Projektes ,,Versorgungsatlas“ (OBSAN 2020). Das OBSAN stellt fest, dass ausgewählte Behandlungen im gemeinsamen Gesundheitsraum deutlich häufiger in Anspruch genommen werden als - im Vergleich zur übrigen Schweiz - zu erwarten gewesen wären. Für die Versorgungsplanung im gemeinsamen Gesundheitsraum ist diese Analyse von hoher Relevanz. Sollten diese Erkenntnisse auch für andere Spitalleistungsbereiche zutreffen, ist davon auszugehen, dass ein Teil der hohen Inanspruchnahme nicht medizinisch begründbar ist. Der Umfang der für diese Leistungen vorgehaltenen Spitalressourcen wäre somit nicht bedarfsgerecht.

Anhand einer vertieften statistischen Analyse wird diese Frage geklärt: Für einzelne Spitalleistungsbereiche muss von einer Überinanspruchnahme in der gemeinsamen Gesundheitsregion ausgegangen werden, die medizinisch nicht begründbar ist. Dies betrifft - unter konservativen Annahmen - 16 Spitalleistungsgruppen mit planbaren Eingriffen u. a. in den Bereichen Bewegungsapparat, HNO, Augenheilkunde, aber auch in der Kardiologie.

\section{- Bedarfsgerechte Nachfrage - Methodische Umsetzung}

Im Rahmen des Projekts wird wie folgt vorgegangen: Wie in der Literatur (zum Beispiel Cutler und Sheiner 1999; Augurzky et al. 2013) werden schrittweise zusätzliche Blöcke von Variablen zur Kontrolle der nachfrageseitigen Erklärung der regionalen Variation in der Inanspruchnahme eingebaut, und zwar:

1. demographische Variablen,

2. Variablen zum Gesundheitszustand und

3. sozioökonomische Variablen.

Interessanterweise zeigen zum Beispiel $\mathrm{Au}-$ gurzky et al. (2013) sowie Zuckerman et al. (2010), dass die bezüglich der oben genannten Kriterien mutmaßlich „umstrittensten“ sozioökonomischen Variablen wie Einkommen, Vermögen usw. relativ wenig zur Reduktion der regionalen Varianz beitragen. Falls dies für die Schweiz ebenfalls gilt, wäre das Problem der „Überkontrolle“ nicht weiter dramatisch. 
$\mathrm{Ob}$ dieser empirische Fall auf Ebene der Inanspruchnahme innerhalb einzelner Leistungsgruppen ebenfalls gilt, wird die Untersuchung zeigen.

Entsprechend werden Kontrollvariablen ausgewählt, welche die regionale Varianz erklären helfen. Die Anzahl der Fälle wird auf diese potenziellen Erklärungsfaktoren regressiert. Da keine Variablen im Modell sind, die das Angebot reflektieren, kann man das Residuum als Indiz für Über- oder Unterversorgung interpretieren (Steiner et al. 2019).

\section{- Bedarfsgerechte Nachfrage}

im gemeinsamen Gesundheitsraum

für das Jahr 2018 und die Folgejahre

Im Rahmen der Standardisierung werden vier aufeinander aufbauende Modelle geschätzt: Ein Modell mit ausschließlich demographischen Variablen (Spezifikation 1); ein Modell mit demographischen Variablen und Variablen zum Gesundheitszustand (Spezifikation 2), ein Modell mit Variablen von Spezifikation 2 plus Variablen zum Bildungsstand und der Haushaltsgröße inklusive der Anzahl der Singlehaushalte (Spezifikation 3); in Spezifikation 4 werden zusätzlich zu den Variablen von Spezifikation 3 noch Variablen zum Einkommen, zur Einkommensungleichheit, zum Vermögen und zur Arbeitslosigkeit eingebaut.

Als favorisierte Version wird die Spezifikation 3 genutzt, welche die potenzielle Überversorgung am besten isoliert und alle drei Einflussfaktoren (Demographie, Gesundheit, Soziodemographie) mit einer relativ geringen Anzahl Kontrollvariablen abbildet. Damit wird auf Variablen verzichtet - mit Ausnahme der Bildungsvariablen -, die nur auf kantonaler Ebene verfügbar sind. Für die Bildungsvariablen wird aufgrund des in der Literatur erwähnten Zusammenhangs (zum Beispiel BFS 2017) auf kantonale Variablen zurückgegriffen. Als Schätzmethode wird eine für Zähldaten passende Methode, die Poisson-Regression, verwendet.

Aus dem Pool von 40 Spitalleistungsgruppen, für die ein Überversorgungspotenzial von mindestens 15 Fällen identifiziert wird, kann für 16 Spitalleistungsgruppen (mit positivem unerklärtem Residuum) aus medizinischer Sicht eine angebotsinduzierte Überversorgung abgeleitet werden. Die defensive Annahme gründet sich auf die Ausführungen des BAG im Jahr 2013 zur „Strategie 2020“ (BAG 2013) sowie den Bericht der Expertengruppe aus dem Jahr 2017 „Kostendämpfungsmassnahmen zur Entlastung der obligatorischen Krankenpflegeversicherung", welche die Relevanz der akutsomatischen Versorgung im Rahmen der Kostendämpfung darstellen. ${ }^{5}$ Auch international verweist der Sachverständigenrat für das Gesundheitswesen (SVR 2018) auf vergleichbare Schätzgrößen.

In Zahlen bedeutet dies (• Tab. 12.1): Nach Standardisierung zeigt sich ein Reduktionspotenzial von 6.643 Fällen $(8,6 \%$ der Gesamtfallzahl) für die 16 Spitalleistungsgruppen. Im Maximalszenario ist davon auszugehen, dass die Abweichung vom erwarteten Wert - angesichts der soziodemographischen Struktur - für die ausgewählten Spitalleistungsgruppen $\mathrm{zu} 100 \%$ angebotsinduziert ist. Für das Versorgungsszenario wird davon ausgegangen, dass im Durchschnitt über alle 16 SPLG rund $50 \%$ der unerklärten Varianz angebotsinduziert sind. Somit wird im Versorgungsszenario von einer bedarfsgerechten Leistungsmenge für das Jahr 2018 ausgegangen, die um 3.321 Fälle (4,3\% der Gesamtfallzahl) geringer ist als im Status quo.

Für die spitalplanungsrelevanten Folgejahre hat dies zur Konsequenz, dass die erwarteten Fallzahlen auf Grundlage der Bedarfsprognose einen Niveaueffekt erfahren (siehe nächsten Abschnitt).

5 Eine Zusammenstellung aller möglichen Effizienzreserven findet sich im Papier Effizienz, Nutzung und Finanzierung des Gesundheitswesens der Akademien der Wissenschaften Schweiz (SAMW 2012). Eine Studie von Polynomics und Helsana, die im Auftrag des BAG durchgeführt wurde, zeigt ein Einsparpotenzial von 10-50\% auf (Brüngger et al. 2014). 
- Tabelle 12.1 Unerklärte Varianz von stationären Behandlungen in der GGR-Bevölkerung nach Spitalplanungsleistungsgruppen - hier nur positive Varianz > 15 Fälle; Kinder bleiben unberücksichtigt (2019)

\begin{tabular}{|c|c|c|c|c|c|}
\hline SPLG & $\begin{array}{l}\text { SPLG-Beschrei- } \\
\text { bung }\end{array}$ & $\begin{array}{l}\text { Unerklärte } \\
\text { Varianz in } \\
\text { Fällen (2016) }\end{array}$ & $\begin{array}{l}\text { Unerklärte } \\
\text { Varianz in } \\
\text { Fällen (2018) }\end{array}$ & $\begin{array}{l}\text { Angebotsinduziert: } \\
50 \% \text { der uner- } \\
\text { klärten Varianz (in } \\
\text { Fällen 2018) }\end{array}$ & $\begin{array}{l}\text { Angebotsinduziert: } \\
50 \% \text { der unerklär- } \\
\text { ten Varianz }\end{array}$ \\
\hline BEW1 & $\begin{array}{l}\text { Chirurgie Bewe- } \\
\text { gungsapparat }\end{array}$ & 712 & 691 & 345 & $11 \%$ \\
\hline BEW2 & Orthopädie & 198 & 185 & 92 & $11 \%$ \\
\hline BEW3 & Handchirurgie & 166 & 189 & 94 & $11 \%$ \\
\hline BEW4 & $\begin{array}{l}\text { Arthroskopie der } \\
\text { Schulter und des } \\
\text { Ellbogens }\end{array}$ & 22 & - & - & $0 \%$ \\
\hline BEW5 & $\begin{array}{l}\text { Arthroskopie des } \\
\text { Knies }\end{array}$ & 841 & 739 & 370 & $17 \%$ \\
\hline BEW6 & $\begin{array}{l}\text { Rekonstruktion } \\
\text { obere Extremität }\end{array}$ & 203 & 171 & 86 & $7 \%$ \\
\hline BEW7 & $\begin{array}{l}\text { Rekonstruktion } \\
\text { untere Extremität }\end{array}$ & 564 & 511 & 255 & $8 \%$ \\
\hline $\mathrm{BP}$ & $\begin{array}{l}\text { Basispaket Chirurgie } \\
\text { und Innere Medizin }\end{array}$ & 3.372 & 3.074 & 1.537 & $5 \%$ \\
\hline GAE1 & Gastroenterologie & 224 & 222 & 111 & $5 \%$ \\
\hline GEF1 & $\begin{array}{l}\text { Gefäßchirurgie } \\
\text { periphere Gefäße } \\
\text { (arteriell) }\end{array}$ & 39 & 26 & 13 & $6 \%$ \\
\hline HNO1 & $\begin{array}{l}\text { Hals-Nasen-Ohren } \\
\text { (HNO-Chirurgie) }\end{array}$ & 269 & 197 & 99 & $10 \%$ \\
\hline HNO1.1 & $\begin{array}{l}\text { Hals- und Gesichts- } \\
\text { chirurgie }\end{array}$ & 60 & 77 & 39 & $9 \%$ \\
\hline HNO1.2 & $\begin{array}{l}\text { Erweiterte } \mathrm{Na}- \\
\text { senchirurgie mit } \\
\text { Nebenhöhlen }\end{array}$ & 144 & 122 & 61 & $8 \%$ \\
\hline KAR1 & $\begin{array}{l}\text { Kardiologie (inkl. } \\
\text { Schrittmacher) }\end{array}$ & 34 & 20 & 10 & $3 \%$ \\
\hline KAR1.1 & $\begin{array}{l}\text { Interventionelle } \\
\text { Kardiologie (Koro- } \\
\text { nareingriffe) }\end{array}$ & 273 & 145 & 72 & $3 \%$ \\
\hline RHE1 & Rheumatologie & 85 & 81 & 40 & $9 \%$ \\
\hline URO1 & $\begin{array}{l}\text { Urologie ohne } \\
\text { Schwerpunkttitel } \\
\text { Operative Urologie }\end{array}$ & 271 & 193 & 96 & $3 \%$ \\
\hline
\end{tabular}

Krankenhaus-Report 2021 
- Die bedarfsgerechten Leistungsmengen in der Zukunft (Prognosen)

Häufig wird eine Prognose mit einer Trendfortschreibung ausgehend von der bisherigen Entwicklung vorgenommen. Dieses Vorgehen setzt aber voraus, dass eine längere Datenreihe ohne größere Änderungen zur Verfügung steht. Dies ist im Gesundheitswesen nicht der Fall.

Aus diesen Gründen ist für Prognosen im Gesundheitswesen ein alternatives Verfahren zu wählen. Dabei werden die oben genannten Einflussfaktoren - soweit machbar - mitberücksichtigt. Da die Entwicklung der Einflussfaktoren nicht präzise vorausgesagt werden kann oder verschiedene Meinungen über die Entwicklung bestehen, werden drei Prognoseszenarien erarbeitet: Szenario „Passiv“, Szenario „Aktiv“, Szenario „Restriktiv“ (- Abb. 12.5).

Das Szenario „Passiv“ beschreibt pro Leistungsgruppe eine Situation, die den Status quo fortschreibt, ohne dass regulierend eingegriffen wird. Im Szenario „Restriktiv“ wird davon ausgegangen, dass das heute bereits bekannte Potenzial ausgeschöpft wird und die Region die zu erwartenden Leistungsmenge erreicht, die im Vergleich zur übrigen Schweiz - nach Standardisierung - zu erwarten wäre; sei es durch ökonomische Anreize oder regulatorische Eingriffe.

Das Szenario „Aktiv“ geht davon aus, dass der normativ definierte Anteil der unerklärten Varianz als angebotsinduzierte Nachfrage ab dem Jahr $\mathrm{t}_{0}$ bis zum Jahr $\mathrm{t}_{+1}$ abgebaut wird (im Szenario „Restriktiv“ wird die gesamte unerklärte Varianz abgebaut). Weiter unterscheiden sich die Szenarien hinsichtlich des Anstiegs der Leistungsinanspruchnahme (Steigung der Graden spiegelt die Trendannahmen wider).

Somit tragen sowohl der Abbau der unerklärten Varianz als auch die zugrunde gelegten Trendannahmen zur Prognose der Leistungsinanspruchnahme bei.

Das Vorgehen im Rahmen des Prognosemodells lässt sich in zwei Phasen gliedern. In der ersten Phase wird auf Grundlage der Bevölkerungsentwicklung der Versorgungsbe-

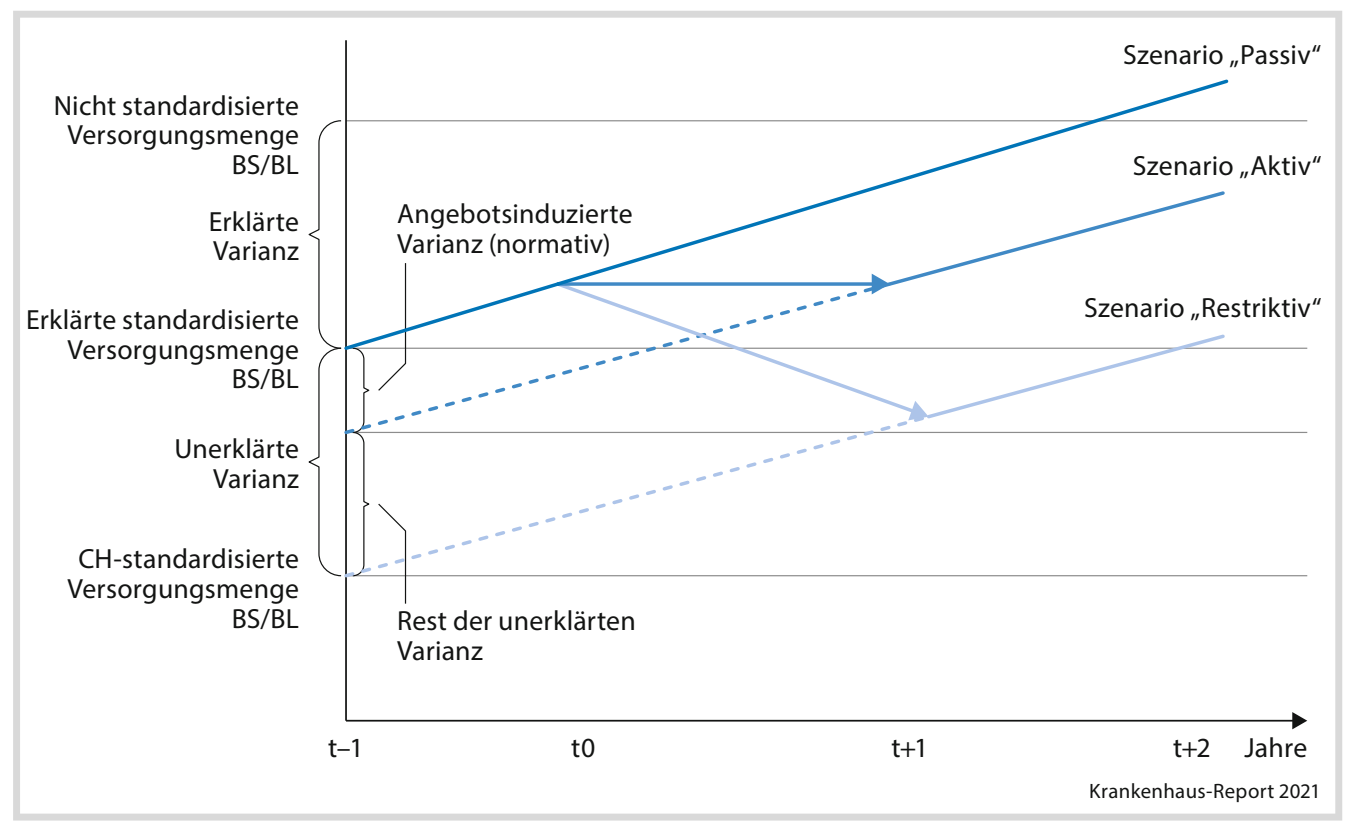

- Abb. 12.5 Entwicklung der Prognoseszenarien 
Ermittlung

- des Versorgungsbedarfs im

Status quo

- der Bevölkerungsentwicklung

Status quo

Prognose

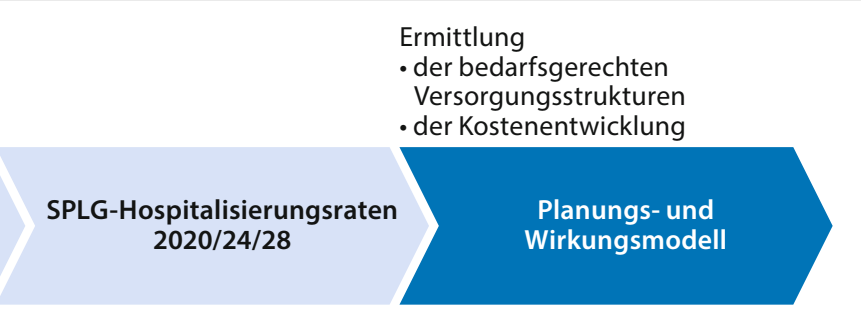

Berücksichtigung von

sozioökonomischen,

epidemiologischen und

medizintechnischen Trends

- Abb. 12.6 Übersicht Prognosemodell

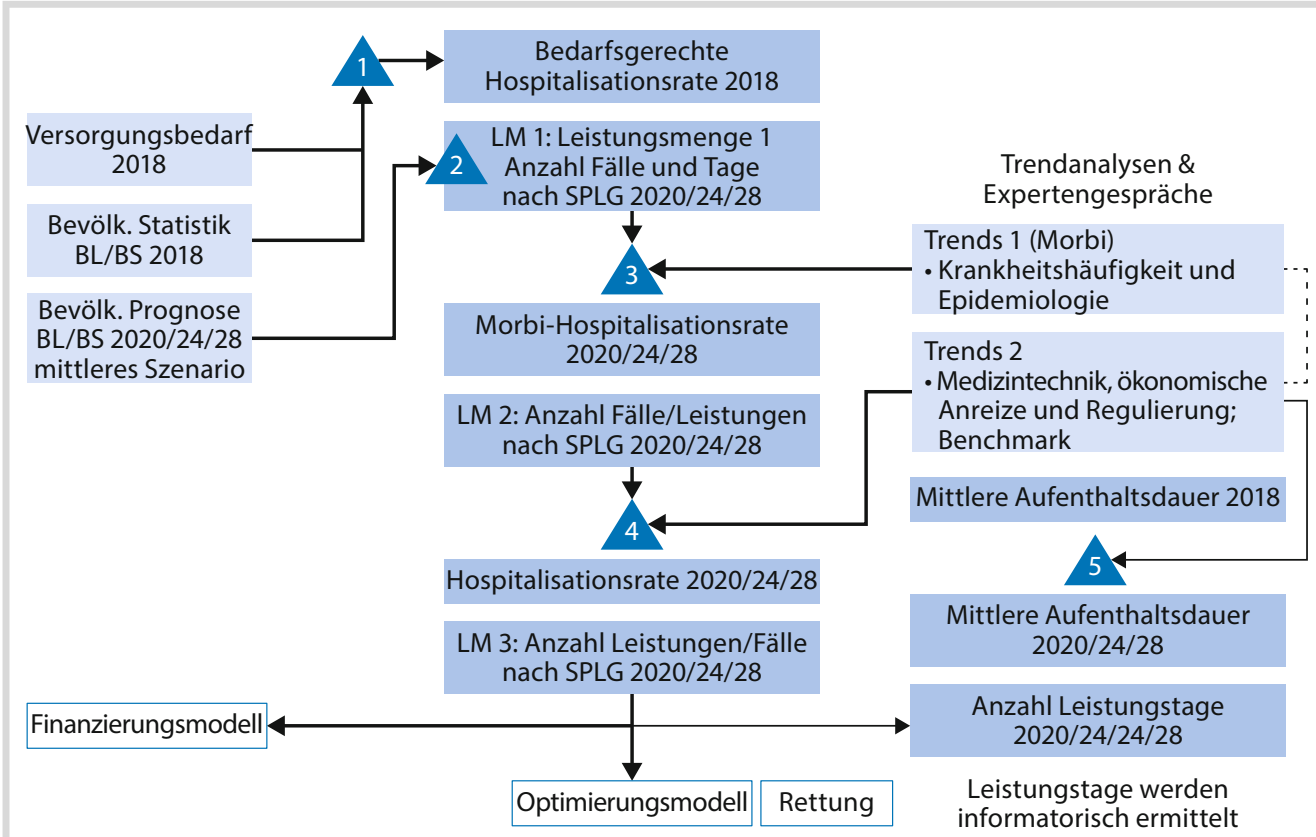

1 Hospitalisierungsrate $2018=$ Fallzahlen (Austritte inkl. ausserkantonal; exkl. Verlegung) auf Grundlage der adjustierten SPLG-Leistungsmenge aus dem Kalenderjahr 2018 / Bevölkerung BS/BL 2018

Leistungsmengen 1 nach SPLG 2020/24/28 (Status quo Leistungsmenge ) =

Hospitalisierungsrate 2018 * Bevölkerung 2020/24/28 (Referenzszenario BFS)

Leistungsmengen 2 = LM 1 korrigiert um epidemiologische und gesellschaftliche Trends

Leistungsmengen 3 = LM 2 korrigiert um ökonomische Anreize und Regulierungsmassnahmen

Zur Ermittlung von Leistungstagen wird die mittlere Aufenthaltsdauer 2018 zugrunde gelegt.

Krankenhaus-Report 2021

- Abb. 12.7 Modell zur Ermittlung der Leistungsdaten (eigene Darstellung in Anlehnung an Gesundheitsdirektion Zürich 2017) 
darf im Status quo in die Zukunft extrapoliert. In der zweiten Phase werden die SPLG-spezifischen Hospitalisierungsraten für die Jahre 2020, 2024 und 2028 unter Berücksichtigung bereits heute identifizierter Trends variiert. Die ermittelten Leistungsmengen fließen unter Berücksichtigung der erwarteten mittleren Aufenthaltsdauer in das Planungs- und Wirkungsmodell ein, um die benötigten Kapazitäten zu ermitteln. Die Wirkungen der Leistungszuteilung auf die Versorgungsstrukturen und Kostenentwicklung werden auf dieser Grundlage analysiert (- Abb. 12.6). Dieser letzte Schritt ist Teil des Prozesses zur Erstellung der Spitallisten.

Das konkrete Vorgehen zur Ermittlung der stationären Leistungsmengen kann wie in - Abb. 12.7 dargestellt skizziert werden.

- Tabelle 12.2 Trendausprägungen

\begin{tabular}{|c|c|c|c|}
\hline Trends & $\begin{array}{l}\text { Szenario „Passiv‘a } \\
\text { (ohne regulatorische } \\
\text { Eingriffe) } \\
\text { Fortschreibung Leis- } \\
\text { tungsinanspruchnahme } \\
\text { Status-Quo }\end{array}$ & $\begin{array}{l}\text { Szenario ,Aktiv“" } \\
\text { (mit maßvollen regulato- } \\
\text { rischen Eingriffen BS/BL) } \\
\text { wahrscheinliche Leis- } \\
\text { tungsinanspruchnahme }\end{array}$ & $\begin{array}{l}\text { Szenario „Restriktiv“6 } \\
\text { (Regulation auf CH- } \\
\text { standardisierten Durch- } \\
\text { schnitt) } \\
\text { zurückhaltende Leis- } \\
\text { tungsinanspruchnahme }\end{array}$ \\
\hline Demographie/Struktur & Referenzszenario $^{\mathrm{b}}$ & Referenzszenario & Tiefes Szenario \\
\hline $\begin{array}{l}\text { Medizintechnik/Digitali- } \\
\text { sierung }\end{array}$ & Ausweitung & Kaum Ausweitung & Keine Ausweitung \\
\hline Epidemiologie & Hohe Leistungszunahme & Mittlere Zunahme & Geringe Zunahme \\
\hline Soziale Trends & Keine Veränderung & Keine Veränderung & Keine Veränderung \\
\hline Ökonomie & Fehlanreize wie bisher & $\begin{array}{l}\text { Fehlanreize teilweise berei- } \\
\text { nigt }\end{array}$ & $\begin{array}{l}\text { Fehlanreize weitgehend } \\
\text { beseitigt }\end{array}$ \\
\hline Regulation & $\begin{array}{l}\text { Keine Substitution „Ambu- } \\
\text { lant vor Stationär““ }\end{array}$ & $\begin{array}{l}\text { 13er-Liste (maximal } 80 \% \\
\text { der bisherigen Leistungen } \\
\text { werden bei unter } 70 \text {-Jäh- } \\
\text { rigen in den ambulanten } \\
\text { Bereich umgeschichtet) } \\
\text { Erweiterte 16er-Liste ab } \\
2021^{c}\end{array}$ & $\begin{array}{l}\text { Erweiterte Liste mit } \\
80 \% \text {-Realisierung in der } \\
\text { Gesamtbevölkerung }\end{array}$ \\
\hline
\end{tabular}

a Lesebeispiel Szenario „Passiv“: Die Inanspruchnahme von Spitalleistungen durch die Bevölkerung folgt dem Trend der letzten Jahre und steigt kontinuierlich an. Weiterentwicklungen in der Medizintechnik werden komplementär zu bestehenden Technologien eingeführt. Für die epidemiologischen Trends wird der „Worst Case“ zugrunde gelegt. Regulatorische Eingriffe erfolgen nicht. Bestehende Fehlanreize, die zu einer Ausweitung der Leistungsinanspruchnahme und Fehlallokationen führen, werden nicht korrigiert (bspw. Vergütung an der Schnittstelle von ambulant zu stationär oder Vergütungsregelungen innerhalb der Spitäler).

b Als Datengrundlage für die demographische Projektion wurden die kantonalen Bevölkerungsszenarien des BFS (2017) verwendet. Das BFS unterscheidet zwischen dem Referenzszenario (Mittleres Szenario) sowie Szenarien mit starkem Bevölkerungswachstum (Hohes Szenario) und geringem Bevölkerungswachstum (Tiefes Szenario). Das tiefe Szenario findet in der vorliegenden Analyse nur im Szenario „Restriktiv“ Anwendung.

${ }^{c}$ Die sogenannte Zürcher-Liste beinhaltet bestimmt Operationen/Eingriffe zu 16 Indikationsfeldern, die primär ambulant durchgeführt werden sollten und nur medizinisch begründeten Fällen stationär erbracht werden. Bis zum Jahr 2021 kommt im Kanton Basel-Stadt eine Liste mit 13 Eingriffen zum Einsatz. Der Kanton Baselland orientiert sich bis dahin an der 6er Liste des Bundes.

Krankenhaus-Report 2021 
- Tabelle 12.3 Annahmen zu Veränderung der Stellgrößen je Prognoseszenario

\begin{tabular}{|l|l|l|l|}
\hline Stellgrößen & $\begin{array}{l}\text { Szenario ,Passiv“ } \\
\text { (ohne regulatorische } \\
\text { Eingriffe) } \\
\text { Fortschreibung Leis- } \\
\text { tungsinanspruchnahme } \\
\text { Status quo }\end{array}$ & $\begin{array}{l}\text { Szenario,Aktiv“6 } \\
\text { (mit regulatorischen Ein- } \\
\text { griffen BS/BL) } \\
\text { Wahrscheinliche Leis- } \\
\text { tungsinanspruchnahme }\end{array}$ & $\begin{array}{l}\text { Szenario,,Restriktiv“6 } \\
\text { (Regulation auf un- } \\
\text { standardisierten CH- } \\
\text { Durchschnitt) } \\
\text { Zurückhaltende Leis- } \\
\text { tungsinanspruchnahme }\end{array}$ \\
\hline $\begin{array}{l}\text { Bevölkerungszahl } \\
\text { Hospitalisierungsrate }\end{array}$ & $\begin{array}{l}\text { Referenzszenario } \\
\text { Kaum Veränderung (nur } \\
\text { Epidemiologie und Medi- } \\
\text { zintechnik) }\end{array}$ & $\begin{array}{l}\text { Mittlere Reduktion, Ermitt- } \\
\text { lung pro SPLG }\end{array}$ & $\begin{array}{l}\text { Starke Reduktion, Ermitt- } \\
\text { lung pro SPLG }\end{array}$ \\
\hline $\begin{array}{l}\text { Mittlere Aufenthalts- } \\
\text { dauer (MAHD) }\end{array}$ & Keine Veränderung & Keine Veränderung & Keine Veränderung \\
\hline Krankenhaus-Report 2021 & & & \\
\hline
\end{tabular}

Die bedarfsgerechte Leistungsmenge (pro SPLG) ist die, um den Korrekturfaktor (angebotsinduzierte Nachfrage) adjustierte Leistungsmenge aus dem Datensatz der medizinischen Statistik. Diese bildet die Basis des Prognosemodells.

Mithilfe der demografischen Daten können für das Jahr 2018 die Hospitalisierungsraten nach Alter und SPLG berechnet werden. Diese werden anschließend auf die aktuellsten Bevölkerungsprognosen angewandt, um die Anzahl der bis zum Jahr 2028 prognostizieren Fälle zu erhalten.

Wie die in - Abb. 12.7 aufgeführten Trends und Stellgrößen bestimmt werden, stellt der folgende Abschnitt dar.

\section{- - Trends und Stellgrößen}

Mit den beschriebenen Prognosevarianten öffnen wir den Wirkungsraum, der für die regionalen Szenarien zur Verfügung steht. Beispielhaft stellt $\bullet$ Tab. 12.2 die Ausprägung der Trends im Prognosezeitraum bis zum Jahr 2028 vor. Die ausführliche Beschreibung der Trends findet sich in den folgenden $\mathrm{Ab}$ schnitten.

Die vorgestellten Trends wirken sich auf die Stellgrößen im Modell aus. Dabei kann kein 1:1-Zusammenhang hergestellt werden. Vielmehr geht es darum, die Annahmen zur
Veränderung der Stellgrößen über die Trends zu validieren.

- Tab. 12.3 stellt dar, welche Veränderungen der Stellgrößen im Prognosemodell bis zum Jahr 2028 vorstellbar sind.

Mit dem präferierten Szenario „Aktiv“ wird eine differenzierte Vorgehensweise gewählt, welche die unterschiedlichen Entwicklungen über die Trends für jeden Leistungsbereich berücksichtigt.

\subsubsection{Was bedeutet diese Erkenntnis für die gemeinsame Versorgungsplanung in der Akutsomatik?}

Die Überversorgung, die weder durch demographische noch durch sozioökonomische Faktoren (wie Bildungsgrad oder Haushaltsgröße) erklärt werden kann, stellt für die Kantonsbevölkerung als Prämien- und Steuerzahlende eine hohe Belastung dar. Jede medizinisch nicht notwendige Operation, die vermieden wird, vermeidet Belastungen für die Patienten und Kosten für die Allgemeinheit. Die Einsparungen werden sowohl die Krankenversicherer entlasten als auch die Ausgaben der 
- Tabelle 12.4 Erwartete Fallzahlentwicklung in Spitalleistungsgruppen mit Mengendialog im Jahr 2024

\begin{tabular}{|c|c|c|c|}
\hline SPLG & $\begin{array}{l}\text { Bezeichnung der Spitalplanungs- } \\
\text { leistungsgruppe }\end{array}$ & $\begin{array}{l}\text { Veränderung } \\
\text { der DRG-Fall- } \\
\text { zahl 2020-2024 }\end{array}$ & Begründung Rückgang \\
\hline ANG1 & Interventionen periphere Gefäße (arteriell) & a & $\begin{array}{l}\text { Umsetzung ,Ambulant vor } \\
\text { Stationär“(AvoS) }\end{array}$ \\
\hline ANG2 & Interventionen intraabdominale Gefäße & a & Umsetzung AvoS \\
\hline AUG1.4 & Katarakt & a & Umsetzung AvoS \\
\hline AUG1.5 & Glaskörper/Netzhautprobleme & a & Umsetzung AvoS \\
\hline BEW1 & Chirurgie Bewegungsapparat & b & $\begin{array}{l}\text { Umsetzung AvoS + Abbau } \\
\text { Überversorgung }\end{array}$ \\
\hline BEW2 & Orthopädie & $\mathrm{b}$ & $\begin{array}{l}\text { Umsetzung AvoS + Abbau } \\
\text { Überversorgung }\end{array}$ \\
\hline BEW3 & Handchirurgie & b & $\begin{array}{l}\text { Umsetzung AvoS + Abbau } \\
\text { Überversorgung }\end{array}$ \\
\hline BEW4 & Arthroskopie der Schulter und des Ellbogens & a & $\begin{array}{l}\text { Umsetzung AvoS + Abbau } \\
\text { Überversorgung }\end{array}$ \\
\hline BEW5 & Arthroskopie des Knies & b & $\begin{array}{l}\text { Umsetzung AvoS + Abbau } \\
\text { Überversorgung }\end{array}$ \\
\hline BEW7 & Rekonstruktion untere Extremität & a & $\begin{array}{l}\text { Umsetzung AvoS + Abbau } \\
\text { Überversorgung }\end{array}$ \\
\hline BP & Basispaket Chirurgie und Innere Medizin & a & $\begin{array}{l}\text { Umsetzung AvoS + Abbau } \\
\text { Überversorgung }\end{array}$ \\
\hline GEF1 & Gefäßchirurgie periphere Gefäße (arteriell) & $\mathrm{a}$ & Abbau Überversorgung \\
\hline GYN1 & Gynäkologie & b & Umsetzung AvoS \\
\hline HNO1 & Hals-Nasen-Ohren (HNO-Chirurgie) & a & $\begin{array}{l}\text { Umsetzung AvoS + Abbau } \\
\text { Überversorgung }\end{array}$ \\
\hline HNO1.1 & Hals- und Gesichtschirurgie & a & Abbau Überversorgung \\
\hline HNO1.2 & Erweiterte Nasenchirurgie mit Nebenhöhlen & a & $\begin{array}{l}\text { Umsetzung AvoS + Abbau } \\
\text { Überversorgung }\end{array}$ \\
\hline HNO1.3.1 & $\begin{array}{l}\text { Erweiterte Ohrchirurgie mit Innenohr und/oder } \\
\text { Duraeröffnung }\end{array}$ & a & Sehr geringe Fahlzahl \\
\hline KAR1 & Kardiologie (inkl. Schrittmacher) & a & $\begin{array}{l}\text { Umsetzung AvoS + Abbau } \\
\text { Überversorgung }\end{array}$ \\
\hline KAR1.1 & Interventionelle Kardiologie (Koronareingriffe) & b & $\begin{array}{l}\text { Umsetzung AvoS + Abbau } \\
\text { Überversorgung }\end{array}$ \\
\hline RHE1 & Rheumatologie & a & $\begin{array}{l}\text { Umsetzung AvoS + Abbau } \\
\text { Überversorgung }\end{array}$ \\
\hline \multicolumn{4}{|c|}{$\begin{array}{l}\text { a Fallzahlrückgang zwischen } 3 \text { bis } 10 \% \\
{ }^{\text {b }} \text { Fallzahlrückgang um mehr als } 10 \% \\
\text { Krankenhaus-Report } 2021\end{array}$} \\
\hline
\end{tabular}


Kantone für Spitalleistungen reduzieren. Immerhin übernehmen die beiden Kantone mindestens $55 \%$ der Kosten für stationäre Spitalaufenthalte. Aufgabe der Versorgungsplanung ist es daher auch, die angebotsinduzierte Überversorgung zu definieren, die im Rahmen der anstehenden Ausgestaltung der gleichlautenden Spitallisten der Kantone Basel-Stadt und Basel-Landschaft im Jahr 2021 reduziert bzw. abgebaut werden kann, ohne die Versorgung der Bevölkerung einzuschränken.

Für die Spitalplanung im Rahmen der Erstellung gleichlautender Spitallisten 2021 sind die ermittelten DRG-Fallzahlen und die Veränderungsraten von hoher Bedeutung. Diese zeigen deutlich auf, dass trotz des Bevölkerungswachstums in einigen Leistungsgruppen mit einem Rückgang der akutsomatischen Fallzahlen bei bedarfsorientierter Planung zu rechnen ist (- Tab. 12.4). Eine Reduktion der Leistungsmenge um rund 4.000 Fälle (im Jahr 2024 gegenüber dem Jahr 2018) entspricht einem jährlichen Brutto-Einsparvolumen $^{6}$ von rund $\mathrm{CHF} 35$ Mio. in der stationären Versorgung des GGR gegenüber der erwarteten Leistungsmenge für das Jahr 2024.

\subsubsection{Das bedarfsorientierte Planungsmodell - technische Umsetzung}

Um der Komplexität der Spitalplanung Rechnung tragen zu können, haben das Amt für Gesundheit des Kantons Baselland (BL) sowie das Gesundheitsdepartement des Kantons Basel-Stadt (BS) ein Planungsmodell erstellen lassen, das die bedarfsgerechten Leistungsmengen auf die Bewerberspitäler unter

a) Berücksichtigung der Interdependenzen der Zürcher Spitalleistungsgruppensystematik und

b) dem Nutzenbeitrag der Spitäler zur Versorgungszielerreichung

6 Das Nettoeinsparvolumen verringert sich um die möglichen Ausgaben in der ambulanten Versorgung (bspw. ambulante OPs, konservative Therapien). verteilt. ${ }^{7}$ Das grundlegende Kriterium für die Verteilung der bedarfsgerechten Leistungsmenge ist der definierte effizienzgewichtete Patienten- und Systemnutzen (ePUS). Die Bedarfsmengen sollen demnach zunächst durch die Spitäler gedeckt werden, die in der jeweiligen Spitalleistungsgruppe den höchsten ePUS aufweisen. Die Verteilung der Bedarfsmengen wird wiederum durch verschiedene definierte Nebenbedingungen limitiert, namentlich die Kapazitäten der Spitäler, die Mindestfallzahlen nach SPLG-Systematik, die verknüpften Leistungen nach SPLG-Systematik sowie Mindestmarktanteile als Kriterium der Versorgungsrelevanz. Die einzelnen Nebenbedingungen werden im Kasten $>$ Nebenbedingungen bei der Verteilung der Bedarfsmengen erklärt und ihre technische Implementierung erläutert.

Methodisch entspricht das Vorgehen einer linearen Optimierung unter (linearen) Nebenbedingungen. Durch das Kriterium der Mindestfallzahl ist der Lösungsbereich insofern eingeschränkt, als die zugeteilten Mengen in Mindestfallzahl-SPLG entweder 0 sind oder mindestens der Mindestfallzahl entsprechen müssen. Zur Lösung eines solchen Problems bieten sich sogenannte Mixed-Integer-LinearProgramming-Algorithmen an. Über das Paket $\mathrm{OMPR}^{8}$ können diese in $\mathrm{R}$ implementiert und somit über eine Shiny-App von außen gesteuert werden.

\section{- Die Ermittlung des Nutzenbeitrags}

Das grundlegende Kriterium für die Verteilung der bedarfsgerechten Leistungsmenge ist der definierte effizienzgewichtete Patienten- und Systemnutzen (ePUS). Der Nutzenbeitrag des Spitals zur Leistungserbringung pro Spitalleistungsgruppe wird in einem gestuften Verfahren

7 Die methodisch-technische Umsetzung des Planungsmodells erfolgt durch das Statistische Amt des Kantons Basel-Stadt. Besonderer Dank gilt Dr. Tobias Erhardt und Dr. Matthias Minke (Statistisches Amt Basel-Stadt 2019) für die fachlich-methodische Unterstützung.

8 https://cran.r-project.org/web/packages/ompr/index. html. 
Nebenbedingungen bei der Verteilung der Bedarfsmengen

\section{Bedarfsdeckung}

$B_{l} \geq \sum_{h=1}^{H} x_{l, h}, \quad$ für alle $l$

Diese Nebenbedingung besagt, dass die Summe der von allen Spitälern $H$ innerhalb einer Leistungsgruppe $l$ zugeteilten Mengen den Bedarf $B_{l}$ nicht übersteigen darf. Die Nebenbedingung wurde bewusst so gewählt, dass eine Unterdeckung des Bedarfs eine zulässige Lösung ist. Dies ermöglicht die Lösbarkeit des Modells in fast allen Konstellationen, die sich aus den restlichen Nebenbedingungen ergeben können. Andersherum führt die strikt steigende Zielfunktion dazu, dass die Bedarfsmenge in einer SPLG immer voll ausgeschöpft wird, wenn dies mit den Nebenbedingungen vereinbar ist.

\section{Kapazitätsbeschränkung}

Die Kapazitätsrestriktion ist in zwei Varianten implementiert, die sich jeweils über die WebApplikation aktivieren lassen.

a. Kapazitäten auf SPLG-Ebene:

$$
\begin{array}{r}
\text { Kapazität }_{h, l} \geq \text { Leistungsmenge }_{h, l} \\
\text { für alle } h \text { und } l
\end{array}
$$

Die Kapazitätsnebenbedingung stellt sicher, dass in keiner Leistungsgruppe ein Spital mehr Fälle zugeteilt bekommt als für dieses Spital für diese SPLG an Kapazität hinterlegt ist.

b. Kapazitäten auf SpitalleistungsbereichsEbene

Ein Spitalleistungsbereich ist eine Zusammenfassung von verschiedenen SPLG, die von der Art der Leistung zusammenhängen. Zum Beispiel bilden die SPLGs BEW1 bis BEW10 den Leistungsbereich „Bewegungsapparat chirurgisch“ Die Kapazitäts- beschränkung auf Spitalleistungsbereichsebene ist demnach so definiert:

$$
\text { Kapazität }_{h, B} \geq \text { Leistungsmenge }_{h, B}
$$$$
\text { für alle } h \text { und } B
$$

Wobei $B$ den Leistungsbereich definiert. Die Aktivierung dieser Restriktion hat zur Folge, dass das Modell die Kapazitäten eines Spitals innerhalb des Leistungsbereichs frei verteilen kann, d. h. die zugeteilten Mengen können die auf SPLG-Ebene definierten Kapazitäten in Einzelfällen überschreiten (zu Lasten der Zuteilung innerhalb einer anderen SPLG).

\section{Mindestfallzahlen (MFZ)}

Durch den Auftraggeber wurden SPLG-spezifische Mindestfallzahlen (MFZ) definiert. Die Idee ist, dass wenn ein Spital innerhalb einer MFZ-SPLG eine Menge zugeteilt bekommt, diese mindestens der Mindestfallzahl entspricht. Diese Anforderung lässt sich mathematisch durch die Einführung von Binärvariablen $b$ lösen, die den Wert 0 annehmen, wenn die Menge 0 beträgt und 1 wenn die Menge positiv ist. Die Mindestfallzahl lässt sich dann über die Definition von zwei Restriktionen mithilfe der sogenannten Big-M-Methode einführen

$m_{l} b_{l, h} \leq$ Leistungsmenge $_{h, l}$ für alle $h$ und $l$ Leistungsmenge $_{h, l} \leq M l_{b, h}$ für alle $h$ und $l$

Die erste Restriktion stellt sicher, dass die zugeteilte Leistungsmenge nur positiv $(b=1)$ sein kann, wenn diese größer ist als die Mindestfallzahl $\mathrm{m}$. Die zweite Restriktion garantiert, dass die Leistungsmenge 0 beträgt, wenn $b=0$. Der Wert für $M$ muss hoch genug gewählt sein, damit es zu keinem Widerspruch mit der Bedarfsdeckung kommt. 


\section{Verbundene Leistungen}

Diese Nebenbedingung stellt sicher, dass Leistungsmengen so zugeteilt werden, dass einige Leistungsgruppen nur zusammen mit fachlich zusammenhängenden Leistungsgruppen verteilt werden können. Einer Leistungsgruppe können eine oder mehrere weitere Leistungsgruppen zugeordnet sein. Wenn mehr als zwei Leistungsgruppen verknüpft sind, so kann diese Verknüpfung auf zwei Arten definiert sein: 1) Eine Leistungsgruppe darf einem Spital nur zugeteilt werden, wenn auch mindestens eine der verknüpften Leistungsgruppen erteilt wird (sog. oder-Verknüpfung). 2) Eine Leistungsgruppe darf einem Spital nur zugeteilt werden, wenn alle der verknüpften Leistungsgruppen erteilt werden (sog. und-Verknüpfung). Die verbundenen Leistungen werden über eine ähnliche Restriktion wie Mindestfallzahl ins Modell eingeführt.

Das Grundprinzip sei hier an einem Beispiel mit der SPLG BEW4, die mit den SPLGs BEW1, BEW2 und BEW3 verknüpft ist, dargelegt. In diesem Fall genügt es, dass eine der weiteren Leistungsgruppen zugeteilt wird (oder-Verknüpfung).

Die Restriktion, die sicherstellt, dass eine Menge für BEW4 nur zugeteilt wird, wenn mindestens auch eine Menge in den Bereichen BEW1, BEW2 oder BEW3 zugeteilt wird, lautet:

$$
\begin{aligned}
& \text { Leistungsmenge }_{h, \mathrm{BEW} 4} \\
& \leq M\left(b_{h, \mathrm{BEW} 1}+b_{h, \mathrm{BEW} 2}+b_{h, \mathrm{BEW} 3}\right) \\
& \text { für alle } h
\end{aligned}
$$

Aus dieser Restriktion folgt, dass die Leistungsmenge für Spital $h$ in der SPLG BEW4 nur positive sein kann, wenn mindestens eine der Leistungsmengen aus BEW1, BEW2 und BEW3 positiv ist (zur Erinnerung: $b$ kann nur 1 sein, wenn die Menge der zugehörigen SPLG positiv ist.)

Erläuterung:

BEW Spitalleistungsbereich Bewegungsapparat

BEW1 Spitalleistungsgruppe: Chirurgie Bewegungsapparat

BEW2 Spitalleistungsgruppe: Orthopädie

BEW3 Spitalleistungsgruppe: Handchirurgie

BEW4 Spitalleistungsgruppe: Arthroskopie der Schulter und des Ellenbogens

\section{Versorgungsrelevanz}

Diese Bedingung besagt, dass ein Spital nur die Leistungsmenge zugeteilt bekommt, wenn es in einer Leistungsgruppe einen Mindestanteil zur Bedarfsdeckung beiträgt.

Leistungsmenge $_{h, l} \geq v_{l} B_{l} b_{h, l} \quad$ für alle $h$ und $l$

$v_{l}$ ist der als Versorgungsrelevanz definierte Mindestanteil an der Bedarfsdeckung. Diese Restriktion greift nur, wenn die zugeteilte Menge überhaupt positiv $\left(b_{h, l}=1\right)$ ist. ermittelt (• Abb. 12.8). Auf der ersten Stufe werden die übergeordneten politischen Ziele

- Ziel 1: eine optimierte Gesundheitsversorgung der Bevölkerung der beiden Kantone

- Ziel 2: eine deutliche Dämpfung des Kostenwachstums im Spitalbereich sowie

- Ziel 3: eine langfristige Sicherung der Hochschulmedizin in der Region operationalisiert und der Nutzenbeitrag der bewerbenden Spitäler pro übergeordnetes Ziel ermittelt. Dies bedeutet, dass die Versorgungsplanung nicht eindimensional den Versorgungsraum anschaut. Die Versorgungsplanung achtet darauf, dass die Ziele gleichgewichtig erreicht werden können. Nur wie soll das gelingen? Die Lösung ist, dass die übergeordne- 


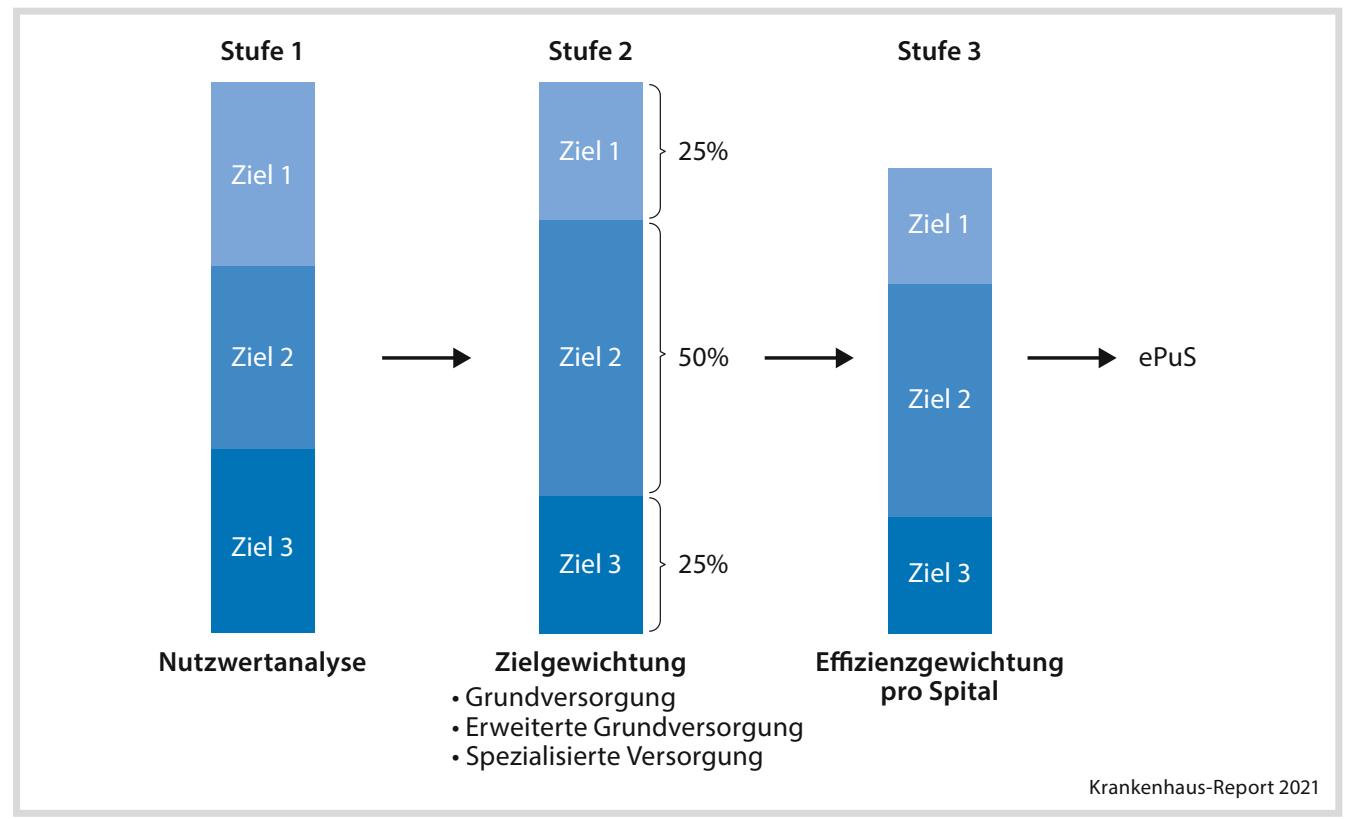

- Abb. 12.8 Drei Stufen zu Ermittlung des Patienten- und Systemnutzens (ePuS)

ten Ziele im weiteren Prozess operationalisiert werden, um den Nutzenbeitrag des jeweiligen Spitals ermitteln zu können. Dabei werden Unterziele definiert, deren Erreichung messbar ist.

Zu Ziel 1 (nicht abschließend) - In einer Region, in der die Menschen neben dem städtischen Zentrum noch in einer eher ländlich geprägten Peripherie leben, spielt die Erreichbarkeit der Grundversorgung/Notfallversorgung eine wichtige Rolle. Dies lässt sich über die Vorgaben zur zeitlichen Erreichbarkeit der Spitalangebote bemessen. Aber auch die Qualität der Versorgung ist ein wichtiges Kriterium.

Zu Ziel 2 (nicht abschließend) - Eine Dämpfung des Kostenwachstums im Spitalbereich schafft den Spielraum dafür, dass die Prämien in der Region Basel in Zukunft weniger stark ansteigen. Neben der preisgünstigen und wirtschaftlichen Leistungserbringung (messbar in Baserate und schweregradbereinigten Fallkosten) spielt auch die Investitionsfähigkeit in effizienzsteigernde Maßnahmen eine wichtige Rolle. Dies drückt sich u. a. in der Eigenkapitalquote sowie der Kennziffer für die operative Leistungsfähigkeit vor Investitionen (EBITDAR-Marge) aus.

Zu Ziel 3 (nicht abschließend) - Ist das Spital Teil eines universitären Netzwerks und beteiligt sich spürbar an der ärztlichen Weiterbildung, ist davon auszugehen, dass es einen Beitrag zur Sicherung der universitären Medizin in der Region leistet.

Im Rahmen einer Nutzwertanalyse (Westermann und Finger 2012; Zangenmeister 1976) wird so der Beitrag des Spitals zur Erreichung der gleichgewichteten übergeordneten Ziele der gemeinsamen Gesundheitsregion bestimmt.

Auf der nächsten Stufe werden die Spitalplanungsleistungsgruppen (SPLG) der Grundversorgung, der erweiterten Grundversorgung oder der spezialisierten Versorgung zugeordnet. Je Versorgungsebenen sind die Ziele unterschiedlich zu gewichten. So wird der Nutzenbeitrag aus Ziel 2 für elektive Spitalplanungsleistungsgruppen der ,erweiterten 
Grundversorgung" höher gewichtet als für Leistungen der Grundversorgung.

Auf der abschließenden dritten Stufe wird der Nutzen eines Spitals pro SPLG einer Effizienzgewichtung unterzogen. Spitäler, die Behandlungen für komplexere multimorbide $\mathrm{Pa}-$ tienten kostengünstig durchführen, erhalten einen Effizienzaufschlag und vice versa (siehe in - Abb. 12.8 ein Beispiel für einen ,Effizienzabschlag“"). Dem liegt aus Sicht der Kantone die Annahme zugrunde, dass die Spitäler, die multimorbide Patienten bei niedrigen Fallausgaben behandeln, Patienten relativ effizient versorgen.

Der Effizienzaufschlag wird durch die den Kantonen zur Verfügung stehenden Kostendaten der Spitäler (CMI, PCCL und Baserate) pro SPLG ermittelt. Im Ergebnis erzielt jedes Spital einen individuellen effizienzgewichteten Patienten- und Systemnutzen pro Spitalplanungsleistungsgruppe (SPLG), der die Verteilung der bedarfsgerechten Leistungsmenge mitbestimmt.

\section{- Eine differenzierte Spitalliste auf Basis von Leistungsmengen}

Mit der Zuteilung von Leistungsmengen durch das Planungsmodell erhält das Spital im Planungsmodell auch einen Leistungsauftrag für die jeweilige Spitalleistungsgruppe. Diese technische Zuteilung muss anschließend durch die langjährige Planungsexpertise der Fachbereiche in beiden Direktionen validiert werden. In wenigen Einzelfällen kommt es zu einer Übersteuerung des technischen Modells, da nicht alle medizinischen Zusammenhänge abschließend in der Zürcher Leistungsgruppensystematik abgebildet werden (können). Diese Abweichungen müssen rechtssicher dokumentiert werden. Im Rahmen der rechtlichen Anhörung haben die Spitäler die Möglichkeit, auf Unklarheiten in der Leistungszuteilung hinzuweisen.
- - Leistungsdialog für Spitalleistungsgruppen mit Tendenz zur Überversorgung

Die in - Tab. 12.1 aufgeführten Spitalleistungen unterliegen dem Mengendialog. Dies bedeutet, dass im Rahmen der Bedarfsanalyse Leistungsbereiche identifiziert wurden, in denen die erbrachten Leistungsmengen nicht dem Bedarf entsprechen (Überversorgung). Die Überversorgung lässt sich zu einem wichtigen Anteil auf Unterschiede in der Ausübung der medizinischen Praxis (medical practice) in den Regionen zurückführen. Die Herausforderung besteht darin, die Überversorgung auf ein bedarfsgerechtes Niveau zu senken.

Die durch eine Bedarfsanalyse ermittelte bedarfsgerechte Leistungsmenge soll als Grundlage einer Zielvorgabe für die Spitäler im GGR dienen. Diese Zielvorgabe wird im Rahmen der gleichlautenden Spitallisten auf die Leistungsaufträge heruntergebrochen. Dies bedeutet, dass die zu vergebenden Leistungsaufträge an maximale Leistungsmengen/ Budgets pro Leistungsauftrag geknüpft werden. Die Zielvereinbarungen werden für ausgewählte Leistungsbereiche in die Leistungsvereinbarungen der Spitäler (Verträge) aufgenommen.

Bei einer drohenden Überschreitung der GGR-Zielvorgabe werden die Spitäler mit entsprechendem Leistungsauftrag von den Kantonen informiert. So besteht die Möglichkeit, dass die Spitäler sich im Vorfeld der Zielvereinbarungsgespräche koordinieren und eigene Maßnahmenvorschläge zur Zielerreichung präsentieren (Mengendialog). Sollten die Vorschläge die Überschreitung der Zielvorgabe nicht verhindern, haben die Regulatoren die Möglichkeit zu reagieren - beginnend mit einem Indikationscontrolling bis hin zum Entzug von Leistungsaufträgen. 


\subsection{Das Planungsmodell setzt die Leitplanken für die Spitalliste}

Mit dem Planungsmodell der beiden Basler Kantone wird sowohl den politischen Zielsetzungen als auch den medizinischen Notwendigkeiten, dem Bedarf der Bevölkerung und der Leistungsfähigkeit der Spitäler Rechnung getragen. Diese komplexen Zusammenhänge lassen sich nur durch eine anerkannte Nomenklatur sowie ein leistungsfähiges lineares Optimierungsmodell technisch abbilden.

Ein weiterer Abgleich mit den bestehenden dezentralen Planungstools zeigt ein hohes Maß an Übereinstimmung mit der gelebten Praxis. Die Ergebnisse des Planungsmodells sind die Leitplanken im weiteren Planungsprozess, an der sich Planungsentscheide orientieren. Nur so bleibt sichergestellt, dass der Bedarf der Bevölkerung an Spitalleistungen in Zukunft in allen Leistungsbereichen abgedeckt ist und gleichzeitig eine nutzenoptimierende Verteilung der Leistungsangebote für die Bevölkerung über die Spitallandschaft hinweg erfolgt. Jede begründete Abweichung vom modellierten optimalen Ergebnis reduziert den ermittelten Nutzwert für die Region. Das Modell kann diese begründeten Abweichungen adaptieren und eine neue Verteilung der Leistungen vornehmen.

Eine Verteilung von Leistungsmengen wird nur für die in der Bedarfsanalyse ermittelten Leistungsgruppen (16 SPLG) vorgenommen, für die ein Mengendialog vorgesehen ist. In allen übrigen Spitalplanungsleistungsgruppen führt die Zuteilung von Leistungsmengen (unter Berücksichtigung der Nebenbedingungen) zur Vergabe eines Leistungsauftrags. Ein Mengendialog ist für diese SPLG nicht vorgesehen.

Mit der Transparenz über Leistungsmengen für ausgewählte Spitalplanungsleistungsgruppen sowie einem Prozess zur Zielerreichung beschreiten beide Kantone Neuland. Sollte dieser kooperative Prozess zum Ziel einer bedarfsgerechten Versorgung führen, könnte dies als Beispiel für andere Spitalplanungen dienen.

Für die Krankenhausplanung in Deutschland kann die Umsetzung in den beiden Basler Kantonen Hinweise darauf geben, was die Erfolgsfaktoren einer effizienten Planung sind. Zum einen bietet die SPLG-Nomenklatur die Chance, von einer kapazitätsorientierten Planung zu einer leistungsorientierten Planung zu kommen, die auch medizinische Notwendigkeiten (inklusive Mindestmengen) berücksichtigt. Zum anderen ist es für die Akzeptanz der Krankenhausplanung empfehlenswert, die Regionalität und eine damit verbundene transparente operationalisierte politische Zielsetzung sicherzustellen (demokratische Legitimierung). Dazu braucht es den politischen Willen, in Gesundheitsräumen zu denken und damit auch regulatorische Gestaltungshoheit zu teilen.

\section{Literatur}

Augurzky B, Kopetsch T, Schmitz H (2013) What accounts for the regional differences in the utilisation of hospitals in Germany? Eur J Health Econ 14(4):615-627

BAG - Bundesamt für Gesundheit (2013) Gesundheit 2020. Die gesundheitspolitischen Prioritäten des Bundesrates. Bern 2013. https://www.bag.admin.ch/bag/ de/home/das-bag/aktuell/medienmitteilungen.msgid-47540.html. Zugegriffen: 17. Juli 2020

BAG - Bundesamt für Gesundheit (2020) Krankenversicherung: Prämienvergleich. https:// www.bag.admin.ch/bag/de/home/versicherungen/ krankenversicherung/krankenversicherungversicherte-mit-wohnsitz-in-der-schweiz/praemienkostenbeteiligung/praemienvergleich.html. Zugegriffen: 17. Juli 2020

BFS - Bundesamt für Statistik (2017) Inanspruchnahme von Versorgungsleistungen und Krebsvorsorge nach sozialer Stellung. BFS Aktuell, Bd. 14. BFS - Bundesamt für Statistik, Neuchâtel

Brüngger B, Fischer B, Früh M, Rapold R, Reich O, Telser H, Trottmann M (2014) Koordinationsbedarf leistungsintensiver Patienten. Polynomics, Olten. https:// www.bag.admin.ch/bag/de/home/strategie-undpolitik/nationale-gesundheitspolitik/koordinierteversorgung/patientengruppen-und-schnittstellen- 
koordinierte-versorgung.html. Zugegriffen: 17. Juli 2020

Cutler DM, Sheiner L (1999) The geography of Medicare. Am Econ Rev 89(2):228-233

Gesundheitsdirektion Zürich (2017) Bedarfsprognose Akutsomatik 2015-2025. Gesundheitsdirektion Zürich, Zürich

Gesundheitsdirektion Zürich (2020) SpitalplanungsLeistungsgruppensystematik. https://www.zh.ch/de/ gesundheit/spitaeler-kliniken/spitalplanung.html\# 346364110. Zugegriffen: 17. Juli 2020

GDK - Konferenz der kantonalen Gesundheitsdirektorinnen und -direktoren (2018) Revidierte Empfehlungen zur Spitalplanung. Verabschiedet von der GDK-Plenarversammlung vom 25.5.2018. https://www.gdkcds.ch/de/gesundheitsversorgung/spitaeler/planung/ empfehlungen-zur-spitalplanung. Zugegriffen: 17. Juli 2020

Lehmann H (2011) Vortrag im Rahmen des SwissDRG Forums 2011

MAGS - Ministerium für Gesundheit, Arbeit und Soziales des Landes Nordrhein-Westfalen (2019) Gutachten Krankenhauslandschaft Nordrhein-Westfalen - Kurzfassung. Hausdruckerei MAGS NRW, Düsseldorf

OBSAN - Schweizerisches Gesundheitsobservatorium (2020) Schweizer Atlas der Gesundheitsversorgung. http://www.versorgungsatlas.ch/. Zugegriffen: 17. Juli 2020

SAMW - Schweizerische Akademie der Medizinischen Wissenschaften (2012) Effizienz, Nutzung und Fi- nanzierung des Gesundheitswesens. Akademien der Wissenschaften Schweiz, Bern

Socialdesign (2018) Monitoring der regionalen und überregionalen Patientenströme (2012-2016). Unveröffentlicht. Basel-Stadt, Basel-Landschaft, Solothurn und Aargau

Statistisches Amt Basel-Stadt (2019) Spitalstandorte im gemeinsamen Gesundheitsraum (GGR)

Steiner M, Nigg M, von Allmen T (2019) Versorgungsplanungsbericht 2019 des Gesundheitsdepartements Basel-Stadt und der Volkswirtschafts- und Gesundheitsdirektion Basel-Landschaft, Basel/Liestal

SVR Gesundheit - Sachverständigenrat zur Begutachtung der Entwicklung im Gesundheitswesen (2018) Bedarfsgerechte Steuerung der Gesundheitsversorgung Gutachten 2018. Medizinisch Wissenschaftliche Verlagsgesellschaft, Berlin

Westermann G, Finger S (2012) Kosten-Nutzen-Analyse. Einführung und Fallstudien. E. Schmidt (ESV basics), Berlin

Zangemeister C (1976) Nutzwertanalyse in der Systemtechnik - Eine Methodik zur multidimensionalen Bewertung und Auswahl von Projektalternativen, 4. Aufl. Wittemann, München

Zuckerman S, Waidman T, Berenson R, Hadley J (2010) Clarifying sources of geographic differences in Medicare spending. N Engl J Med 363(1):54-62

Open Access Dieses Kapitel wird unter der Creative Commons Namensnennung 4.0 International Lizenz (http:// creativecommons.org/licenses/by/4.0/deed.de) veröffentlicht, welche die Nutzung, Vervielfältigung, Bearbeitung, Verbreitung und Wiedergabe in jeglichem Medium und Format erlaubt, sofern Sie den/die ursprünglichen Autor(en) und die Quelle ordnungsgemäß nennen, einen Link zur Creative Commons Lizenz beifügen und angeben, ob Änderungen vorgenommen wurden.

Die in diesem Kapitel enthaltenen Bilder und sonstiges Drittmaterial unterliegen ebenfalls der genannten Creative Commons Lizenz, sofern sich aus der Abbildungslegende nichts anderes ergibt. Sofern das betreffende Material nicht unter der genannten Creative Commons Lizenz steht und die betreffende Handlung nicht nach gesetzlichen Vorschriften erlaubt ist, ist für die oben aufgeführten Weiterverwendungen des Materials die Einwilligung des jeweiligen Rechteinhabers einzuholen.

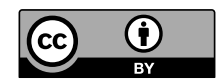

